\title{
Pathological complete response in a patient with locally advanced pancreatic adenocarcinoma treated with neoadjuvant gemcitabine and S-1: a case report and literature review
}

\author{
Surong Hua ${ }^{\#}$ Junyi Gao ${ }^{\# \wedge}$, Qiang Xu, Xiafei Hong, Wenming Wu \\ Department of General Surgery, State Key Laboratory of Complex Severe and Rare Diseases, Peking Union Medical College Hospital, Chinese \\ Academy of Medical Science and Peking Union Medical College, Beijing, China \\ "These authors contributed equally to this work and should be considered as co-first authors. \\ Correspondence to: Wenming Wu, MD. Department of General Surgery, State Key Laboratory of Complex Severe and Rare Diseases, Peking Union \\ Medical College Hospital, Chinese Academy of Medical Science and Peking Union Medical College, Beijing 100730, China. \\ Email: doctorwuu@126.com.
}

\begin{abstract}
Backgroundk Pancreatic ductal adenocarcinoma (PDAC) is one of the most lethal cancers worldwide. Radical resection is currently the only potential curative treatment. However, over $80 \%$ of patients present with unresectable tumor at the time of diagnosis. It is recommended that patients with unresectable pancreatic cancers be offered neoadjuvant treatment. A combination of gemcitabine and S-1 (GS-1) has been reported to be an effective regimen for unresectable pancreatic cancers, however, there have been no reports of pathological complete response up until now.

Case Description: Herein, we present a 67-year-old male who presented with a 4-month history of upper abdominal and back pain, as well as unintentional weight loss. Abdominal computed tomography (CT) confirmed a hypovascular mass in the pancreas neck consistent with unresectable pancreatic cancer. Positron emission tomography (PET)/CT also revealed a high fludeoxyglucose (FDG)-avid lesion in the pancreas neck without evidence of distant metastasis. Pancreatic adenocarcinoma was confirmed with ultrasound-guided fine-needle aspiration cytology. The patient was recommended to undergo treatment with gemcitabine and S-1. After 5 cycles of neoadjuvant chemotherapy, CT and PET/CT both revealed the disappearance of the lesion and a pancreaticoduodenectomy was offered as a potentially curative treatment. Histological assessment revealed no evidence of residual adenocarcinoma [ypT0N0 (0/38)]. The tumor marker cancer antigen (CA)125 increased one month after the surgery, resulting in two additional cycles of GS-1. This patient remained disease-free for 21 months after surgery.

Conclusions: This report is the first to present a case of a pathological complete response in a patient with locally advanced pancreatic cancer following GS-1 treatment, suggesting radical resection after GS-1 chemotherapy might be a potential curative treatment strategy for unresectable PDAC.
\end{abstract}

Keywords: Neoadjuvant therapy; pathological complete response; locally advanced pancreatic adenocarcinoma; case report

Submitted Dec 14, 2021. Accepted for publication Feb 11, 2022.

doi: $10.21037 /$ gs-22-6

View this article at: https://dx.doi.org/10.21037/gs-22-6

^ ORCID: 0000-0002-7214-8448. 


\section{Introduction}

Pancreatic ductal adenocarcinoma (PDAC) is the fourth most lethal malignancy worldwide. Over $80 \%$ of PDAC patients present with unresectable tumors at the time of diagnosis, with almost half of the tumors being locally advanced, invading or even encasing the celiac axis, abdominal aorta, or superior mesenteric artery (1). Recent research has reported on the efficacy of neoadjuvant treatment in pancreatic tumors, and this may have promising effects on surgical excision and quality of life (QoL). Pathologic complete response (pCR) is defined as no viable residual tumor, which is much more anecdotal in pancreatic cancer than in other cancers after neoadjuvant therapies. Herein, we present a case of a patient with locally advanced PDAC who achieved pCR after neoadjuvant treatment with gemcitabine and S-1 (GS-1). We present the following case in accordance with the CARE reporting checklist (available at https://gs.amegroups.com/article/ view/10.21037/gs-22-6/rc).

\section{Case presentation}

A 67-year-old Chinese male presented to Peking Union Medical College Hospital in December 2016, with a 4-month history of upper abdominal and back pain as well as unintentional weight loss. He had a personal history of smoking, and his family history included one brother with pancreatic cancer and another brother with rectal cancer.

Laboratory exams showed normal complete blood count and serum bilirubin. His liver enzymes were slightly elevated, and his amylase (AMY) was $173 \mathrm{U} / \mathrm{L}$ (25-125 U/L). On first visit, his carbohydrate antigen (CA)199 was $1,299 \mathrm{U} / \mathrm{mL}(0-34 \mathrm{U} / \mathrm{mL})$, CA125 was $86.7 \mathrm{U} / \mathrm{mL}$ (reference range, $0-35 \mathrm{U} / \mathrm{mL}$ ), and carcinoembryonic antigen (CEA) was $23.2 \mathrm{ng} / \mathrm{mL}(0-5 \mathrm{ng} / \mathrm{mL})$.

Abdominal enhanced computer tomography (CT) scan revealed a hypovascular mass $(1.86 \mathrm{~cm} \times 1.67 \mathrm{~cm})$ in the pancreas neck, radiographically consistent with PDAC. This mass almost completely encircled the common hepatic artery (CHA), as well as the splenic artery and vein (Figure 1). Multiple retroperitoneal small lymph nodes (LNs) were also observed in the enhanced CT images.

The patient underwent a positron emission tomographycomputed tomography (PET/CT), which revealed a high fludeoxyglucose (FDG)-avid lesion $(3.3 \mathrm{~cm} \times 2.5 \mathrm{~cm} \times 3.9 \mathrm{~cm}$, $\mathrm{SUV}_{\max }$ 10.2) in the pancreas neck (Figure 2), as well as an enlarged LN with increased FDG uptake $\left(\mathrm{SUV}_{\max } 1.5-1.6\right)$, without evidence of distant metastasis. Percutaneous ultrasound (US)-guided biopsy of the lesion showed cytology consistent with PDAC (Figure 3).

The patient was diagnosed with locally advanced, unresectable pancreatic adenocarcinoma (cT4NxM0, stage III) according to the 8th American Joint Committee on Cancer (AJCC) TNM classification. At the time of diagnosis, the patient's body surface area (BSA) was $1.59 \mathrm{~m}^{2}$ and his functionality was consistent with Eastern Cooperative Oncology Group (ECOG) grade 1. It was recommended that the patient receive chemotherapy with gemcitabine (1.4 $\mathrm{g}$ qd on days 1 and 8$)$, combined with S-1 (40 mg qd + $60 \mathrm{mg}$ qn from day 1 to day 14$)$ for each course of treatment. As grade 4 neutropenia was observed during the first course, doses of gemcitabine and S-1 were reduced accordingly. After the second cycle, expression of the tumor markers decreased (Figure 4), and the tumor showed obvious shrinkage (Figure 5). These improvements indicated a partial response (PR) to therapy according to the Response Evaluation Criteria in Solid Tumors (RECIST, version 1.1).

After 5 cycles of chemotherapy, the tumor markers decreased significantly, with CA125 and CEA levels reaching the normal range, and CA19-9 levels were just slightly above the upper normal limits (Figure 4). Enhanced CT images and three-dimensional reconstructed images showed that the tumor had disappeared, and no constriction was found in the celiac trunk, CHA, nor splenic artery (Figure 6). Restaging PET/CT showed no FDG-avid uptake in the neck of the pancreas nor the surrounding structures (Figure 7).

According to the significantly improved CT results and negative FDG-avid uptake on PET-CT, the patient underwent pancreaticoduodenectomy in June 2017. No vascular resection nor reconstruction was required, and the postoperative process was uneventful. Histological assessment of the specimen revealed no evidence of residual adenocarcinoma, and only scattered foci of grade 2 pancreatic intraepithelial neoplasia (PanIN2) were observed (Figure 8). All 38 resected LNs were negative for malignancy, confirming a complete pathological response (ypT0N0).

In the follow-up visit one month after the surgery, the patient's CA125 increased to $161 \mathrm{U} / \mathrm{mL}$ (Figure 4). Two more cycles of GS-1 chemotherapy were considered. The dose of gemcitabine was reasonably reduced as a grade 3 neutropenia was observed after the first cycle. The value of CA125 eventually decreased. Tumor recurrence and metastasis occurred 21 months after surgery based on the 


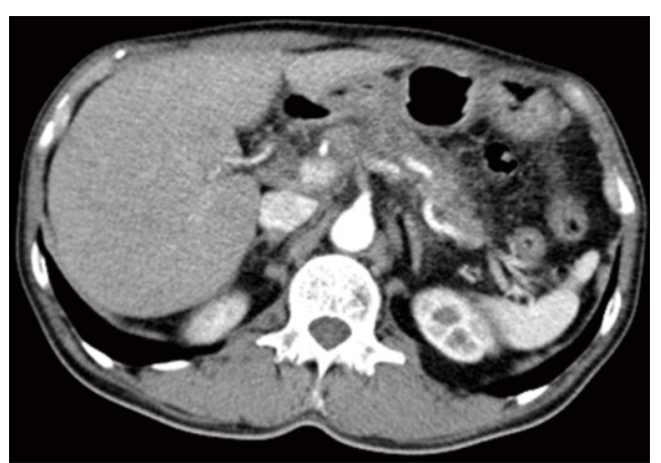

Figure 1 Axial CT demonstrating large mass in the pancreas neck which infiltrated the common hepatic artery, splenic artery and vein before neoadjuvant therapy.

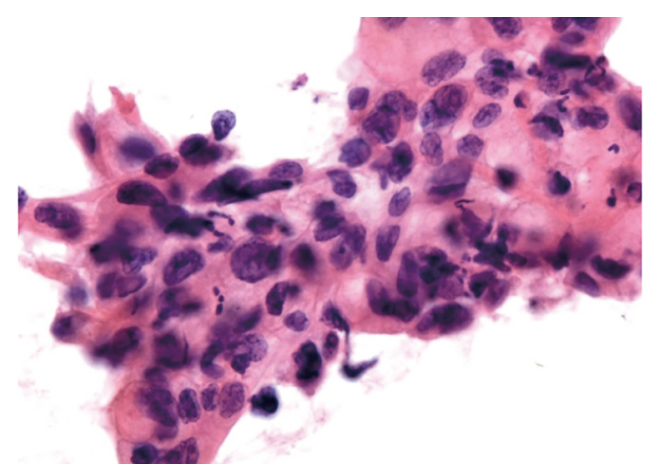

Figure 3 Pancreatic adenocarcinoma cells were found in the bioptic specimen [hematoxylin and eosin (H\&E) stained, 400×].
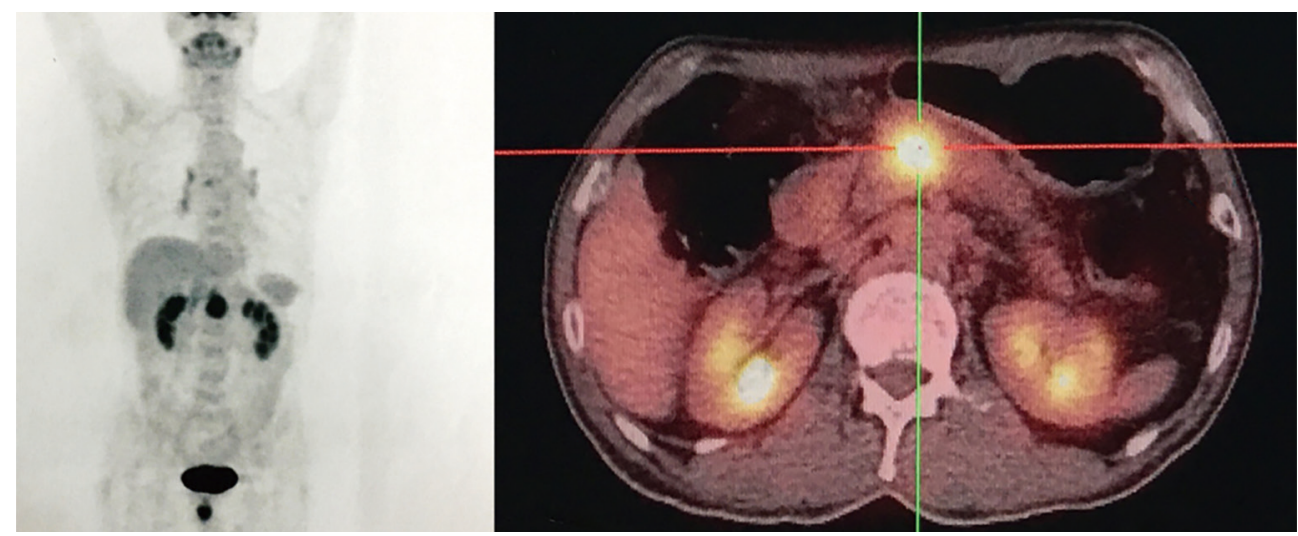

Figure 2 PET/CT images (coronal images on the left, axial images on the right). A FDG-avid uptake lesion in the neck of the pancreas $\left(3.3 \mathrm{~cm} \times 2.5 \mathrm{~cm} \times 3.9 \mathrm{~cm}, \mathrm{SUV}_{\max }\right.$ 10.2). PET/CT, positron emission tomography-computed tomography; FDG, fludeoxyglucose.

results of CT and PET-CT, and the patient was eventually lost to follow-up at 27 months after surgery. All procedures performed in this study were in accordance with the ethical standards of the institutional and/or national research committee(s) and with the Helsinki Declaration (as revised in 2013). Written informed consent was obtained from the patient for publication of this case report and accompanying images. A copy of the written consent is available for review by the editorial office of this journal.

\section{Discussion}

PDAC is one of the most devastating malignancies with only $20-25 \%$ of PDAC patients receiving surgical resection and adjuvant therapy (2). Unfortunately, radical surgery is not possible for most patients due to locally advanced or metastatic lesions. To downstage unresectable lesions and proceed to subsequent surgery, great effort has been invested into exploring neoadjuvant chemotherapy with or without concomitant radiotherapy in patients with pancreatic cancers. The main agents of neoadjuvant chemotherapy for pancreatic cancer nowadays include gemcitabine, 5-fluorouracil (5-FU) and its oral analogues, mitomycin $\mathrm{C}$, and platinum compounds. As monotherapies, both gemcitabine and 5-FU or its oral analogues, have been widely investigated and found to be associated with unsatisfactory outcomes. Recently, more attention has focused on combination therapies, such as FOLFIRINOX (folinic acid, fluorouracil, irinotecan, and oxaliplatin) and gemcitabine-based and 5-FU-based regimens (additional components include mitomycin, cisplatin, oxaliplatin, capecitabine, S1, etc.). However, the efficacy 


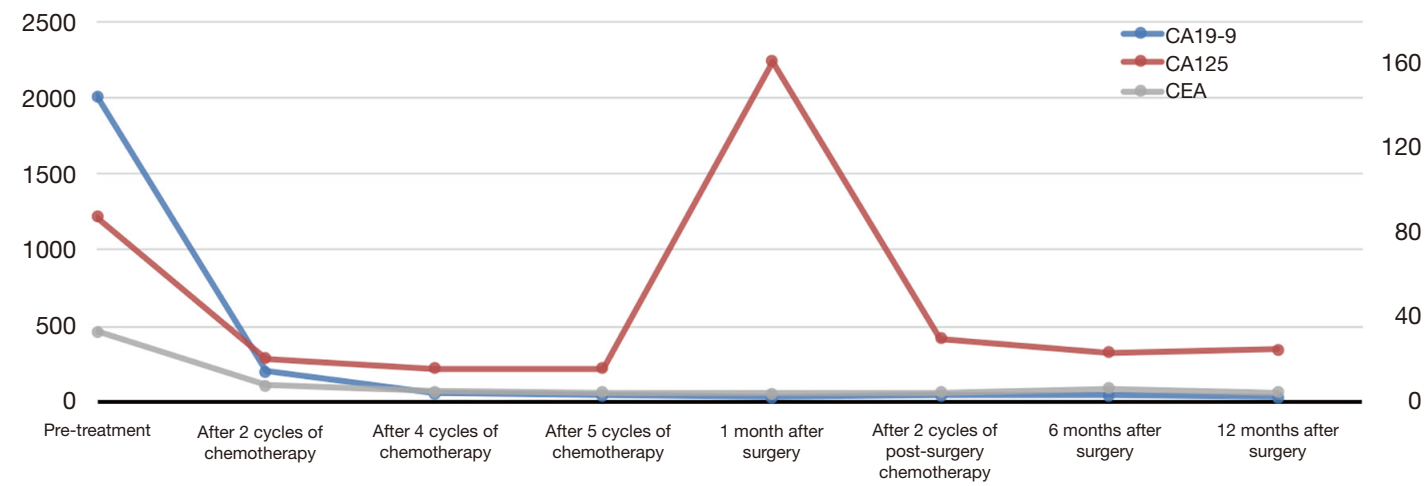

Figure 4 Changes of the values of tumor markers during the management (the left Y-axis represents the value of CA19-9, the right Y-axis represents the value of CA125 and CEA).

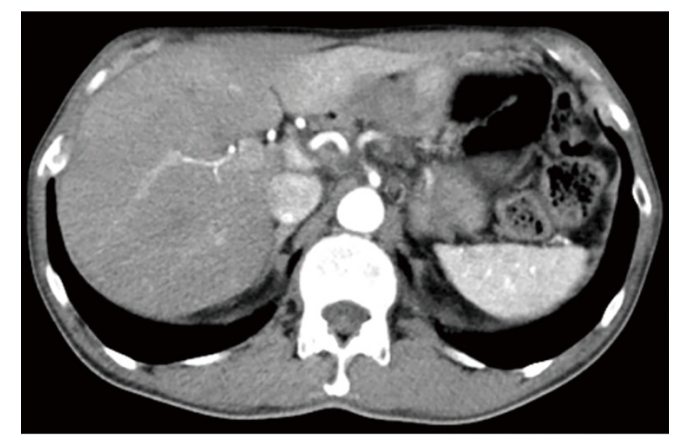

Figure 5 Axial CT demonstrating the mass in the neck of pancreas shrank after 2 cycles of neoadjuvant therapy.

of these treatments is still controversial. Heinemann et al. performed a meta-analysis including 15 randomized trials to compare single-agent gemcitabine to gemcitabine-based two-drug combination chemotherapy, and a significant survival improvement was achieved in gemcitabine + fluoropyrimidines or platinum analogs, while such improvement was not observed in other combination (3). Several prospective phase II trials and database analyses have suggested that there are advantages to neoadjuvant therapy, including tumor downstaging, lower surgical complication rates, improved QoL, and favorable overall survival (OS) (4). Despite neoadjuvant therapy presenting as a promising treatment for pancreatic cancer, there remain some important limitations, and much debate is focused on the safety including toxicities, infections, deterioration of nutritional status and disease progression, as well as on inclusion criteria of neoadjuvant chemotherapy for primarily resectable or borderline resectable tumors (5).

Resectability of localized pancreatic tumor is frequently impeded by the infiltration of adjacent structures, such as celiac arteries and superior mesenteric vessels. As vascular resection is thought to be risky or even impossible for some pancreatic cancer surgeons, the resectability of advanced tumors is extremely dependent on their individual experience and proficiency. Currently, the classification of locally advanced PDAC is consistent, with unresectable tumors defined as lesion abutment over 180 degrees of the mesenteric or hepatic arteries, as well as involving the superior mesenteric vein (SMV) without practicable reconstruction (6).

The initial therapy for locally advanced PDAC is monoor combination systemic chemotherapy. Gemcitabine has obvious advantages in palliating the disease and improving OS, making it the standard treatment for advanced PDAC. The oral fluoropyrimidine S-1 consists of the 5-fluorouracil (5-FU) prodrug tegafur, and two enzyme inhibitors, 5-chloro-2,4-dihydroxypyridine (CDHP) and potassium oxonate $(\mathrm{OXO})$. S-1 was designed with the aim of enhancing the anticancer activity of $5-\mathrm{FU}$, while reducing its gastrointestinal toxicity. A phase 3 trial carried out by Uesaka $e t$ al. investigated the non-inferiority of S-1 to gemcitabine as adjuvant chemotherapy for pancreatic cancer, and results showed the 5 -year OS was $24.4 \%$ vs. $44.1 \%$ (gemcitabine group vs. $\mathrm{S}-1$ group, $\mathrm{P}<0.0001$ ) (7). The efficacy of S-1 has also been confirmed in other solid tumors, including gastric, colorectal, head and neck, breast, and non-small cell lung cancers (8).

Table 1 summarizes the randomized controlled trials (RCTs) and retrospective studies in the literature exploring the efficacy of GS-1 in advanced pancreatic cancer with or without metastasis. 

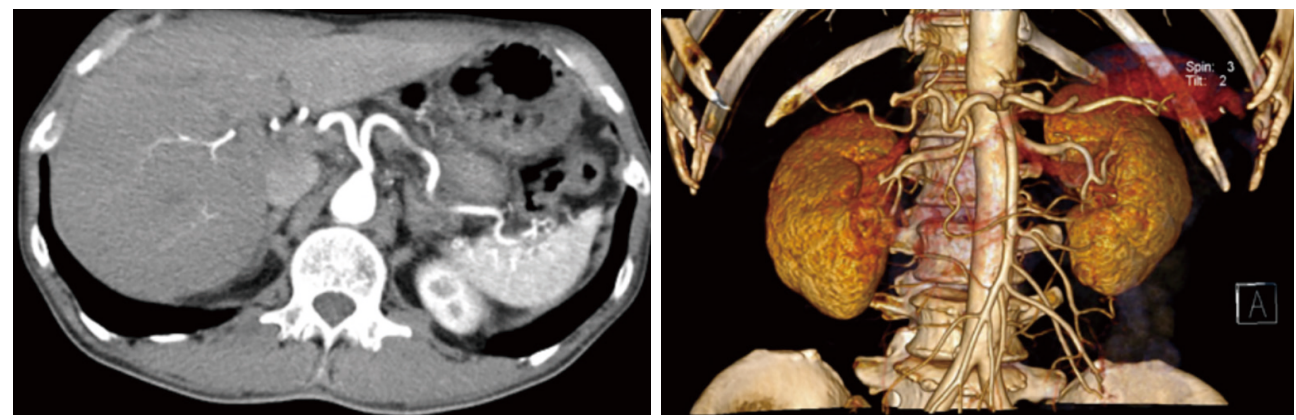

Figure 6 Axial CT (left) showed a little area with low enhancement where the previous tumor located, and in the three-dimensional reconstructed image, no constriction was found in celiac trunk, common hepatic artery and splenic artery after 5 cycles of neoadjuvant therapy.

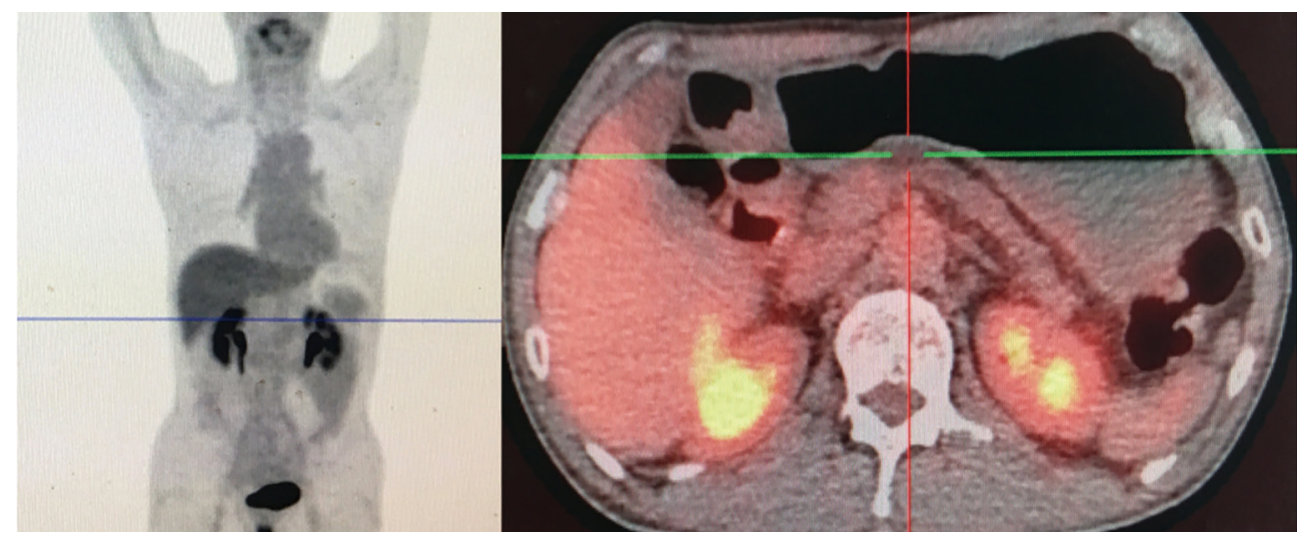

Figure 7 PET/CT images (coronal images on the left, axial images on the right). Absence of FDG-avid uptake in the pancreas after 5 cycles of the neoadjuvant therapy. PET/CT, positron emission tomography-computed tomography; FDG, fludeoxyglucose.

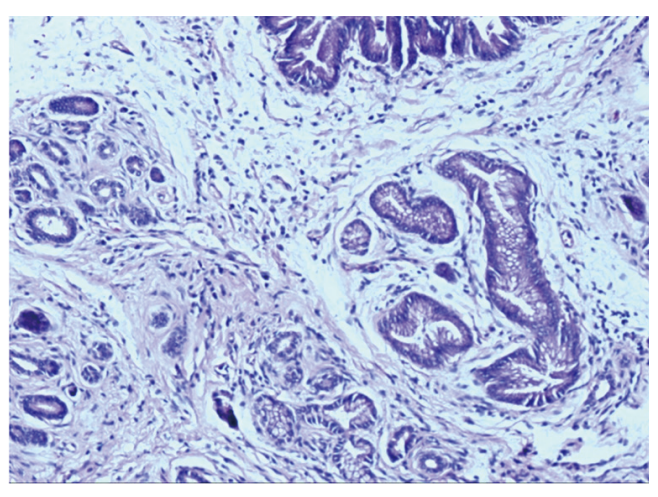

Figure $8 \mathrm{H} \& \mathrm{E}$ sections demonstrating pancreatic tissue with dense fibrosis, residual ducts, and islet cells. No evidence of residual carcinoma $(40 \times)$.
Ueno et al. conducted a phase III clinical trial enrolling 834 patients affected by locally advanced and metastatic pancreatic cancer, with the aim of investigating the noninferiority of S-1 alone and the superiority of GS-1 compared with gemcitabine alone (5). The objective response rate (ORR) and disease control rate (DCR) in the GS-1 group were $29.3 \%$ and $71.5 \%$, respectively, which were significantly higher than the rates observed in the gemcitabine group. Compared with gemcitabine, GS-1 showed an advantage in improving progression free survival (PFS), while it failed to achieve a statistically significant improvement in OS. Imaoka et al. further analyzed the data from elderly patients in the RCT (9). The results showed 


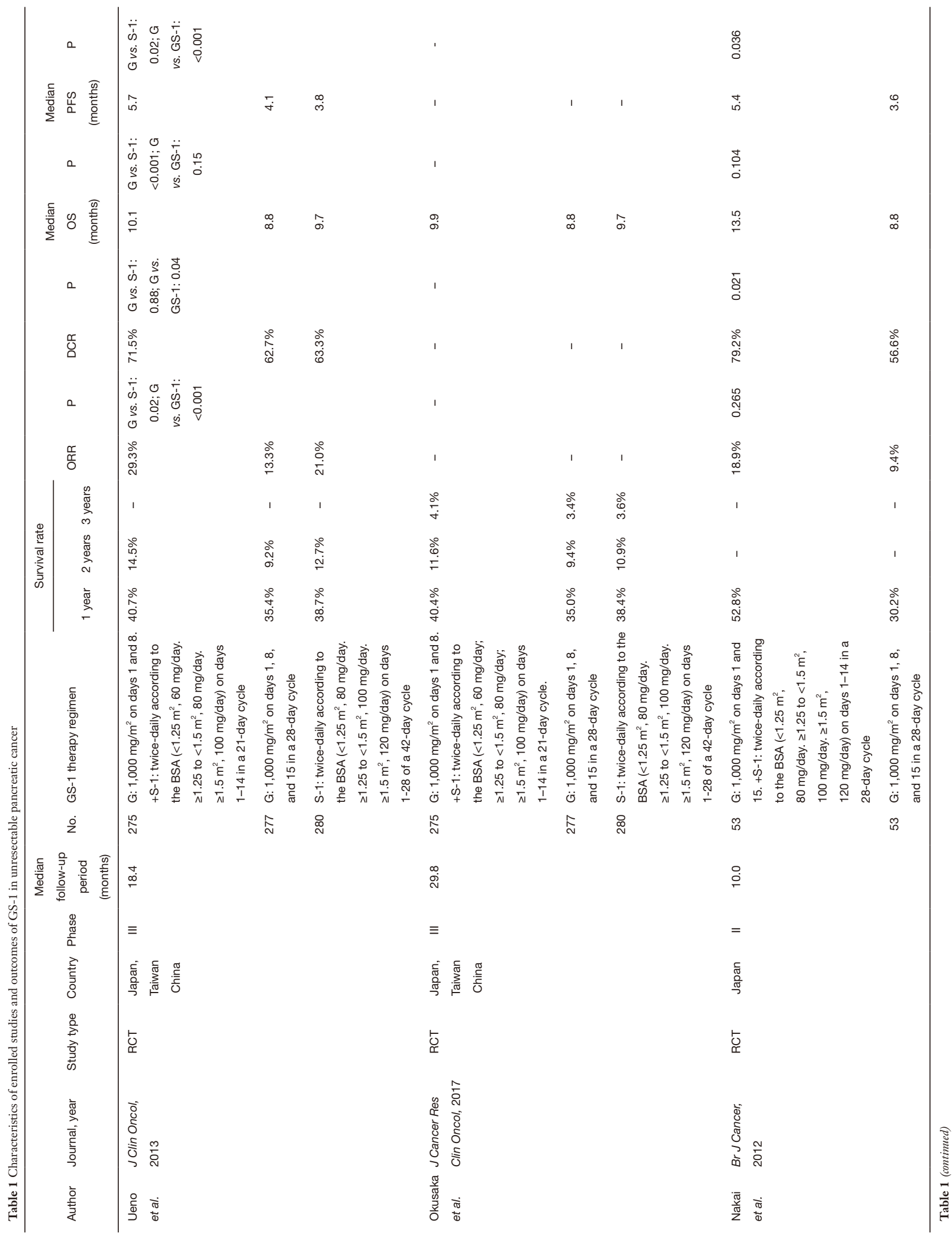




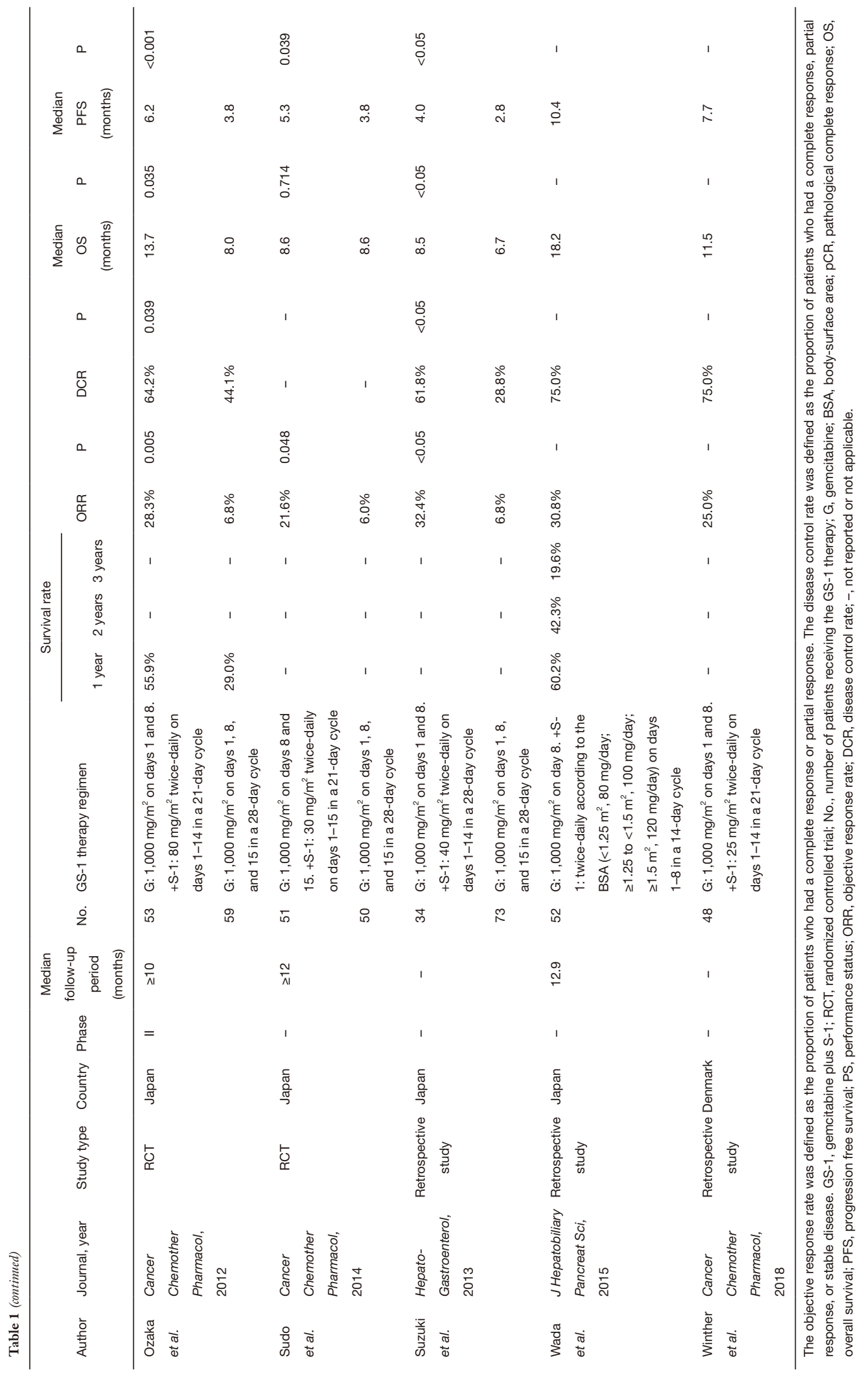


similar objective ORR and OS in the three groups but a higher rate of hematological events in the GS-1 subgroup. Therefore, it was recommended that GS-1 be used with caution as the first-line treatment for elderly patients. To reconfirm the results achieved in the previous RCT and to further explore the efficacy of GS-1, Okusaka et al. prolonged the follow-up period and concluded that S-1 can be used as a standard treatment for advanced pancreatic cancer (10).

Nakai et al. reported a randomized phase II trial comparing the efficacy, safety, and dose intensity between gemcitabine and GS-1 in advanced pancreatic cancer (11). Although a higher one-year survival rate and longer median PFS were demonstrated in the GS-1 group compared with gemcitabine alone, the OS showed no statistical difference. Moreover, further analysis in patients with locally advanced tumors indicated no statistically significant results in median PFS nor OS. Another two RCT studies consistently showed the superiority or noninferiority of GS-1 in terms of ORR, DCR, median PFS, and OS compared to the gemcitabine alone, and similar results were observed in a retrospective study (12-14). However, a higher frequency of adverse events tended to occur in the GS-1 group, and the major grade 3-4 adverse events included neutropenia, thrombocytopenia, and skin rash $(12,14)$.

Wada et al. reported a series of 52 patients affected by locally advanced and metastatic pancreatic cancer treated with biweekly GS-1 (15). This regimen might be an alternative option for unresectable pancreatic cancer, as suggested by the long OS and PSF, high ORR and DCR, and low frequency of severe adverse events with a $5.8 \%$ rate of hematological toxicity.

Although the above studies all suggested that GS-1 has promising clinical benefits, the patients enrolled in these studies were all Asians. Recently, Winther et al. published a breakthrough study exploring the efficacy and toxicity of GS-1 in Caucasian patients with unresectable pancreatic cancer (16). A total of 64 patients were enrolled and 48 received GS-1 as initial therapy. The data indicated that GS-1 was reliable, safe, and showed promising efficacy in the Caucasian population, with an ORR of $25.0 \%$, a DCR of $75.0 \%$, a 7.7 -month median PFS [95\% confidence interval (CI): 6.7 to 8.9 ], and a 11.5-month median OS (95\% CI: 9.7 to 12.3 ). Additionally, the most common adverse event was fatigue (86\%) and grade 3 events only occurred in $3 \%$ of patients. Cao et al. performed a metaanalysis including 4 RCTs to examine the efficacy of GS-1, and results showed a significant OS improvement in locally advanced patients but not in metastatic patients, which suggested its disadvantage in metastatic cases (17).

A pCR indicates the presence of neoplastic tissue without viable invasive cancer cells within the specimen. To date, pCR in pancreatic cancer has always been observed with chemoradiation, rather than chemotherapy alone. Barugola et al. reported that neoadjuvant therapy could lead to a $7 \%$ pCR in patients with borderline resectable or locally advanced PDAC (18). Tatsuguchi et al. reported a case that a 72 -year-old male with far advanced intrahepatic cholangiocarcinoma achieved a pCR after 10 cycles of GS-1 chemotherapy (19). In addition, a patient with advanced gastric cancer was also reported to achieve a pCR after S-1 chemotherapy (20). These cases suggested that GS-1 chemotherapy could be a hopeful frontline treatment for advanced carcinoma. To the best of our knowledge, the patient presented herein is the first case report of a complete pathological response in a patient affected by a locally advanced PDAC treated with GS-1. Indeed, studies in the literature have confirmed that neoadjuvant chemotherapy with GS-1 is safe and can provide a high response rate and long OS in Asian patients, and similar potential benefits have also been observed in the Caucasian population.

Although there have been several studies confirmed the efficacy of GS-1 or S-1 chemotherapy in pancreatic cancer, our manuscript firstly reported a rare outcome of $\mathrm{pCR}$ following GS-1 therapy, suggesting radical resection after GS-1 chemotherapy might be a potential curative treatment strategy for unresectable PDAC. As tumor heterogeneity has a great impact on chemotherapy efficacy, it is very important to select the corresponding chemotherapeutic drugs according to the tumor characteristics of different patients. In the future, drug screening based on the molecular characteristics of patients will become a potential breakthrough in pancreatic cancer treatment.

\section{Acknowledgments}

Funding: This work was supported by the National Natural Science Foundation of China (No. 81902517).

\section{Footnote}

Reporting Checklist: The authors have completed the CARE reporting checklist. Available at https://gs.amegroups.com/ article/view/10.21037/gs-22-6/rc

Conflicts of Interest: All authors have completed the ICMJE 
uniform disclosure form (available at https://gs.amegroups. com/article/view/10.21037/gs-22-6/coif). The authors have no conflicts of interest to declare.

Ethical Statement: The authors are accountable for all aspects of the work in ensuring that questions related to the accuracy or integrity of any part of the work are appropriately investigated and resolved. All procedures performed in this study were in accordance with the ethical standards of the institutional and/or national research committee(s) and with the Helsinki Declaration (as revised in 2013). Written informed consent was obtained from the patient for publication of this case report and accompanying images. A copy of the written consent is available for review by the editorial office of this journal.

Open Access Statement: This is an Open Access article distributed in accordance with the Creative Commons Attribution-NonCommercial-NoDerivs 4.0 International License (CC BY-NC-ND 4.0), which permits the noncommercial replication and distribution of the article with the strict proviso that no changes or edits are made and the original work is properly cited (including links to both the formal publication through the relevant DOI and the license). See: https://creativecommons.org/licenses/by-nc-nd/4.0/.

\section{References}

1. Hidalgo M. Pancreatic cancer. N Engl J Med 2010;362:1605-17.

2. Oettle H, Post $\mathrm{S}$, Neuhaus $\mathrm{P}$, et al. Adjuvant chemotherapy with gemcitabine vs observation in patients undergoing curative-intent resection of pancreatic cancer: a randomized controlled trial. JAMA 2007;297:267-77.

3. Heinemann V, Boeck S, Hinke A, et al. Meta-analysis of randomized trials: evaluation of benefit from gemcitabinebased combination chemotherapy applied in advanced pancreatic cancer. BMC Cancer 2008;8:82.

4. Barenboim A, Lahat G, Geva R, et al. Neoadjuvant FOLFIRINOX for locally advanced and borderline resectable pancreatic cancer: An intention to treat analysis. Eur J Surg Oncol 2018;44:1619-23.

5. Ueno H, Ioka T, Ikeda M, et al. Randomized phase III study of gemcitabine plus S-1, S-1 alone, or gemcitabine alone in patients with locally advanced and metastatic pancreatic cancer in Japan and Taiwan: GEST study. J Clin Oncol 2013;31:1640-8.
6. Heinrich S, Lang H. Neoadjuvant Therapy of Pancreatic Cancer: Definitions and Benefits. Int J Mol Sci 2017;18:1622.

7. Uesaka K, Boku N, Fukutomi A, et al. Adjuvant chemotherapy of S-1 versus gemcitabine for resected pancreatic cancer: a phase 3, open-label, randomised, noninferiority trial (JASPAC 01). Lancet 2016;388:248-57.

8. Saif MW, Syrigos KN, Katirtzoglou NA. S-1: a promising new oral fluoropyrimidine derivative. Expert Opin Investig Drugs 2009;18:335-48.

9. Imaoka H, Kou T, Tanaka M, et al. Clinical outcome of elderly patients with unresectable pancreatic cancer treated with gemcitabine plus S-1, S-1 alone, or gemcitabine alone: Subgroup analysis of a randomised phase III trial, GEST study. Eur J Cancer 2016;54:96-103.

10. Okusaka T, Miyakawa H, Fujii H, et al. Updated results from GEST study: a randomized, three-arm phase III study for advanced pancreatic cancer. J Cancer Res Clin Oncol 2017;143:1053-9.

11. Nakai $Y$, Isayama H, Sasaki T, et al. A multicentre randomised phase II trial of gemcitabine alone vs gemcitabine and S-1 combination therapy in advanced pancreatic cancer: GEMSAP study. Br J Cancer 2012;106:1934-9.

12. Ozaka M, Matsumura $Y$, Ishii H, et al. Randomized phase II study of gemcitabine and S-1 combination versus gemcitabine alone in the treatment of unresectable advanced pancreatic cancer (Japan Clinical Cancer Research Organization PC-01 study). Cancer Chemother Pharmacol 2012;69:1197-204.

13. Suzuki S, Ozaki Y, Saida S, et al. Retrospective study of gemcitabine plus S-1 versus gemcitabine alone in cases with unresectable advanced pancreatic cancer. Hepatogastroenterology 2013;60:916-20.

14. Sudo K, Ishihara T, Hirata N, et al. Randomized controlled study of gemcitabine plus S-1 combination chemotherapy versus gemcitabine for unresectable pancreatic cancer. Cancer Chemother Pharmacol 2014;73:389-96.

15. Wada K, Sano K, Amano H, et al. Biweekly gemcitabine plus S-1 for locally advanced and metastatic pancreatic cancer: a preliminary feasibility study. J Hepatobiliary Pancreat Sci 2015;22:692-8.

16. Winther SB, Bjerregaard JK, Schonnemann KR, et al. S-1 (Teysuno) and gemcitabine in Caucasian patients with unresectable pancreatic adenocarcinoma. Cancer Chemother Pharmacol 2018;81:573-8.

17. Cao C, Kuang M, Xu W, et al. Gemcitabine plus S-1: a hopeful frontline treatment for Asian patients with 
unresectable advanced pancreatic cancer. Jpn J Clin Oncol 2015;45:1122-30.

18. Barugola G, Partelli S, Crippa S, et al. Outcomes after resection of locally advanced or borderline resectable pancreatic cancer after neoadjuvant therapy. Am J Surg 2012;203:132-9.

19. Tatsuguchi T, Gotoh K, Kobayashi S, et al. Pathologic complete response after gemcitabine and S-1 chemotherapy

Cite this article as: Hua S, Gao J, Xu Q, Hong X, Wu W. Pathological complete response in a patient with locally advanced pancreatic adenocarcinoma treated with neoadjuvant gemcitabine and S-1: a case report and literature review. Gland Surg 2022;11(2):494-503. doi: 10.21037/gs-22-6 for far advanced intrahepatic cholangiocarcinoma. Int Cancer Conf J 2018;7:93-7.

20. Namikawa T, Ishida N, Tsuda S, et al. Pathological complete response by $\mathrm{S}-1$ chemotherapy in advanced gastric cancer. In Vivo 2018;32:1211-6.

(English Language Editor: J. Teoh) 